\title{
Integrating Dual Antennas with Hybrid DC32-QAM Modulation to Increase the Coverage Area for Wireless Sensor Networks
}

\author{
Mason Adam, Martin Hope, Haifa Takruri-Rizk \\ The University of Salford, Salford, M5 4WT, United Kingdom \\ m.adam1@edu.salford.ac.uk; m.d.hope@salford.ac.uk; h.takruri-rizk@salford.ac.uk
}

Keywords: Wireless Sensor Networks (WSN), Wireless Personal Area Networks (WPAN), Ultra-Wideband (UWB), Multiple Input Multiple Outputs (MIMO), Dual Carrier Modulation (DCM), DC 32-QAM.

\begin{abstract}
WSN's and WPAN technologies continue to develop rapidly with integration into numerous applications. The ever increasing demands on these systems require that radio transceiver extend their coverage area and adopt more efficient algorithms to full fill the requirements of new applications. In this paper, a unique application of Multiple Input Multiple Output (MIMO) technology is applied to the application of WSN's in order to dynamically extend range and thus overall coverage area. The scheme incorporates multiple antennas with Dual Circular 32-QAM modulation for MB-OFDM based systems in order to increase the wireless communication range. Simulations results are then presented that have shown a significant improvement in the system performance in terms of the bit error rate and also signal to noise power ratio.
\end{abstract}

\section{Introduction}

MIMO technology is a well-established antennae technology that can extend the link coverage area for wireless communication systems [1-4]. By incorporating the spatial diversity of MIMO technology with a Dual Carrier Modulation (DCM) scheme that is specified for data rates of $320 \mathrm{Mbps}, 400 \mathrm{Mbps}$ for systems operating at the Ultra Wide-Band (UWB) domain, it is shown that a substantial improvement in the coverage area is possible. For these types of applications, the modulation and coding schemes naturally require modification. In this context, existing research has considered increasing the number of coded bits associated with each Inverse Fast Fourier Transform (IFFT) sub-carrier tone in the modulation process. In this paper, the authors propose a novel scheme incorporating a modified Dual Circular (DC) 32 - Quadrature Amplitude Modulation (QAM) for Multi-Band Orthogonal Frequency Division Multiplexing (MB-OFDM) based systems specifically in order to dynamically enhance propagation range. The main advantages obtained with this proposal are achieved without the need for additional transmission power which makes it very attractive for wireless communication networks that have limited power reserves [5]. However, whilst there is a limited amount of research available on the implementation 
of MIMO technology to UWB applications. The IEEE have established the IEEE 802.15.3a standards group based on previous research work by Batra et al.[6]. This work was developed in order to define a standard model for the UWB PAN physical layer in order to meet the growing demands for UWB applications with high data rates. Subsequently the IEEE 802.15.3a task group was established which specifies a communication range for UWB systems at less than $10 \mathrm{~m}[7]$.

In this paper, a proposal that integrates MIMO technology for wireless sensor networks based on the WiMedia framework using MB-OFDM is presented. It considers a review of existing work, and then a proposal of a novel approach to extending the coverage area of the wireless communication link. The proposed ECMA368 standard [8] is also considered, and a UWB system with a multiple antenna configuration is presented as a basis for the analysis. The proposed model allows an increase in the coverage area by optimising the received power without violating the Federal Communications Commission (FCC) transmission regulations. In summary this model expands the fading diversity across the modulated symbols within the code-word, and improves the performance of the spatial and frequency domains.

This paper is organised as follows: sections two and three provide general description of the proposed model and a review of the physical channel model that is used to describe the medium response in the UWB domain. Section four provides a review of MIMO multiband modulation schemes used in the licence free spectrum. Section five then provides an analytical description of the principle use of spatial elements in extending wireless propagation range. Section six and seven describe the transmitting and receiving structure of the proposed model. Section eight then presents a simulation and performance analysis for the proposed model. The paper concludes with a summary of this work.

Notation: This paper will use the following notations. Superscript $†$ represnet the the transpose conjugate and \|\|$_{F}$ stands for the Frobenius norm of a vector or matrix. Bold upper case letters and bold and bold lower case letters donate matrices and vectors, respectively. The superscript ${ }^{H}$ stands for element wise conjugation, and $\mathrm{Q}$ (.) refer to the error function.

\section{System Overview}

In wireless sensor networks, the demand for extending wireless range is a critical function of the system and can sometimes become more important than the throughput requirement. In this context, the proposed model can be configured to replace existing multiplexing techniques and fully exploit diversity across the spatial and frequency domains. 
The principle design presented in this paper is based on a hybrid modulation scheme consisting of a combination of two modulation schemes, namely Quadrature Phase Shift Keying (QPSK) and 8 Phase Shift Keying (8PSK) in combination with dual transmitting antennas, to provide overall a DC32-QAM with MIMO configuration. The concept depends on a combination of two layers of modulations based on a core QPSK modulation and an additional sequence of binary bits. In order to accomplish this, the input is interleaved and segmented into two sequences; the first is applied to the Quadrature modulation, while the second sequence is used in the upper dimension of the 8PSK scheme.

There are three phases required to perform this modulation scheme. The first is to carry out QPSK modulation; the second is to modified the modulated symbols in order to induce a new and modified quadrature modulated symbols (QPSK symbols); and the third, is to use these new QPSK symbols with a binary sequence allocated in the upper dimension of the 8PSK constellation map, thus creating equal energy within the 8PSK modulation spread across two antennas.

The novelty of this proposal is to produce a configuration model that make use of the diversity properties along with a combination of two modulation schemes that allows transmitting at higher dimension constellation while decoding with a lower dimension at the receivers. This allows transmitting at higher rate while ensuring larger Euclidean metric between neighbouring signals at the receivers' constellation. Therefore increasing the noise energy requirement to shift the received signals into to the erroneous regions (reducing the noise errors), and hence reducing the error rate of the system. The equal decision region for symbol bits for the Phase shift keying scheme gives this particular modulation an advantage in terms of Peak to Average Power Ratio (PAPR).

\section{Physical Channel Analysis}

In order to validate this work, the proposed system was subjected to a well-known and verified physical channel model. As a result, the proposed DC-32QAM based system was subjected to the IEEE803.15.3a model for validation and verification. Within this standard model (SG3a) the amplitude of the multipath fading reflects the signal distribution at the receiver resulting from the filtering effect of the channel. The characteristic of the received signal was obtained by actual measurements and a stochastic analysis for the indoor environment in the standard. This standardised model uses a sampling period of $0.167 \mathrm{~ns}$ (nanosecond) for the discretisation process of the UWB channel response, translating this continuous response into a discrete sample model. It is important to maintain the properties and percentage ratios of the channel characteristic of this model. Therefore the following parameters were kept consistent with the standards requirements:

The cluster arrival rate, Ray arrival rate, Cluster attenuation constant, Ray attenuation constant, The overall standard deviation of the multipath shadowing with a lognormal distribution, Standard deviation of lognormal 
shadowing for the clusters, Standard deviation of lognormal shadowing for the rays within a particular cluster, The mean excess delay, The number of significant paths within 10dB of the peak power, The power Delay Profile (PDP), and the RMS delay spread.

For simulation purposes, a down conversion with filtering and resampling was undertaken by adjusting the sampling period. This simulation condition was required due to the arbitrary arrival times of the UWB channel and therefore its bandwidth cannot be limited. Resampling resulted in a sampling period of $2.672 \mathrm{~ns}(0.167 * 16)$, where the sampling index was multiplied by sixteen. In order to remove the aliasing effect before the decimation when adjusting the resampling, a code with anti-aliasing filtering or complex down conversion was used.

\section{Review of modulation Schemes}

Dual Carrier Modulation (DCM) has been suggested by Balakrishnan et al. [9] to accommodate the need for higher data rate applications as part of the ECMA standard. It uses frequency diversity by allocating the DCM symbol (which consists of four bits) to two separate subcarriers separated by $200 \mathrm{MHz}$. A total number of 100 complex symbols are then mapped to 100 IFFT (Inverse Fast Furrier Transform) subcarriers in the transmitter block before transmission [10]. The demapping process at the receiver then uses the two constellation maps and combines the subcarriers that represent the same symbol. Each two subcarriers containing the I (In phase) and Q (Quadrature phase) components of that particular symbol are separated by 50 FFT (Fast Furrier Transform) tones. Hence this diversity produces two different signals with various power strengths associated with the symbol [11].

The theoretical data rate of $480 \mathrm{Mbps}$ for the DCM scheme could be hard to maintain in a real channel environment due to the hostile nature of wireless links. The DC 32-QAM scheme was proposed in order to increase the system throughput by mapping more information on each subcarrier of the 100 IFFT data tones [12].

\section{Analytical Analyses}

The analytical analysis is important factor in evaluating the proposed method, and in this work the concept of spatial diversity was used to improve the wireless range. As it is analytical analysis, the error performance will be bounded, and to accurately measure the performance of the system, an upper bound of the error principle was used in this analysis. In order to elaborate further on the concept of using multiple spatial elements in wireless communication, the following analysis was carried out. Assuming the transmitted signals (s) across radiating elements was erroneously detected (e). Then, the error probability $P(E)$ in terms of the error conditional probability $P(E \mid H S)$ based on the stochastic quantity of signal to noise ratio at the output $\beta$ could be expressed as follows

$P(E)=\int_{0}^{\infty} P(E \mid \beta) \quad P(\beta) d \beta$ 
And

$P(E \mid \beta)=Q\left(\sqrt{\frac{E}{N o}} D(\boldsymbol{s}, \boldsymbol{e})\right)$

Where

$D(\boldsymbol{s}, \boldsymbol{e})=\sum_{r=1}^{R} \sum_{n=1}^{N}\left\|\sum_{t=1}^{T} h_{r}^{t} \Delta_{n}^{t}(s, e)\right\|_{F}$

Where $h$ is the channel coefficients, and $\Delta$ is the Euclidean distance metric (Euclidean noise energy between codewords).

Further rearrangement and manipulation leads to

$$
\begin{gathered}
D(\boldsymbol{s}, \boldsymbol{e})=\sum_{r=1}^{R} \sum_{n=1}^{N}\left(\sum_{t_{2}=1}^{T}\left(h_{r, n}^{t_{2}} \Delta_{n}^{t_{2}}(s, e)\right)^{\dagger} \quad \sum_{t_{1}=1}^{T} h_{r, n}^{t_{1}} \Delta_{n}^{t_{1}}(s, e)\right) \\
=\sum_{r=1}^{R} \sum_{t_{2}=1}^{T}\left(\sum_{t_{1}=1}^{T}\left(h_{r}^{t_{2}}\right)^{\dagger} h_{r}^{t_{1}} \sum_{n=1}^{N}\left(\Delta_{n}^{t_{2}}(s, e)\right)^{H} \Delta_{n}^{t_{1}}(s, e)\right)
\end{gathered}
$$

Let assume

$\boldsymbol{K}^{t_{2}, t_{1}}=\sum_{n=1}^{N}\left(\Delta_{n}^{t_{2}}(\boldsymbol{s}, \boldsymbol{e})\right)^{\dagger} \Delta_{n}^{t_{1}}(\boldsymbol{s}, \boldsymbol{e})$

Then, $\boldsymbol{K}^{t_{2}, t_{1}}$ represented a matrix with Harmition property, then applying matrix decomposition results in the following

$\boldsymbol{K}=\boldsymbol{A}^{\dagger} \boldsymbol{\Sigma} \boldsymbol{A}$, and $\boldsymbol{\Sigma}=\sum_{t=1}^{T} \lambda_{t}$

Where $A$ is unitary matrix, and $\Sigma$ is a digonal matrix

Assuming multipath fading with Rayleigh distribution, and applying the Chernoff bound express of the Qfunction on the error formula [13], then the error probability formula becomes

$P(E) \leq \int_{0}^{\infty}|H| \exp \left(\sum_{r=1}^{R} \sum_{t_{2}=1}^{T} \sum_{t_{1}=1}^{T}\left(h_{r}^{t_{2}}\right)^{H} h_{r}^{t_{1}} A^{t_{2}, t_{1}} \Sigma\left(A^{t_{2}, t_{1}}\right)^{*}\right) \exp \left(-|H|^{2}\right) d H$

Let $\varphi=h_{r}^{t_{1}} A^{t_{2}, t_{1}}$, that lead to

$P(E) \leq \int_{0}^{\infty}|\mathrm{H}| \exp \left(\sqrt{\frac{E_{x}}{2 N_{0} N_{t}}} \sum_{r=1}^{R} \sum_{t=1}^{T} \lambda_{t} \phi_{t}^{r}\right) \exp \left(-|H|^{2}\right) d H$

$P(E) \leq \int_{t=1}^{T}|\mathrm{H}| \prod_{r=1}^{R} \prod_{t=1}^{T} \exp \left(\sqrt{\frac{E_{x}}{2 N_{0} N_{t}}} \lambda_{t} \phi_{t}^{r}\right) \exp \left(-|H|^{2}\right) d H$

$P(E) \leq \int_{t=1}^{T}|\mathrm{H}| \prod_{r=1}^{R} \prod_{t=1}^{T} \exp \left(-\sqrt{\frac{E_{x}}{2 N_{0} N_{t}}} \lambda_{t} \phi_{t}^{r}-|H|^{2}\right) d H$ 


$$
\begin{aligned}
P(E) \leq & \int_{0}^{\infty}|\mathrm{H}| \prod_{r=1}^{R} \prod_{t=1}^{T} \exp -\left(\sqrt{\frac{E_{x}}{2 N_{0} N_{t}}} \lambda_{t} \phi_{t}^{r}+1\right)|H|^{2} d H \\
& \operatorname{Let}\left(\sqrt{\frac{E_{x}}{2 N_{0} N_{t}}} \lambda_{t} \phi_{t}^{r}+1\right)=\Psi
\end{aligned}
$$

Then, inserting this term to the formula results in the following

$$
P(E) \leq \int_{0}^{\infty}|\mathrm{H}| \prod_{r=1}^{R} \prod_{t=1}^{T} \exp -(\Psi)|H|^{2} \quad d H(4)
$$

Introducing the following Laplace transforms expression [14] to (4)

$$
\int_{0}^{\infty} \exp ^{-s y} d y=\frac{1}{s}, s>0
$$

Then, the probability of error could be derived as follows

$$
P(E)=\prod_{r=1}^{R} \prod_{t=1}^{T} \frac{1}{\Psi}=\prod_{r=1}^{R} \prod_{t=1}^{T} \frac{1}{\left(\sqrt{\frac{E_{x}}{2 N_{0} N_{t}}} \lambda_{t} \phi_{t}^{r}+1\right)}
$$

From the equation of (5), one can detect that by increasing the number of radiating elements, the probability of error will be reduced. In addition, the received signal power will be optimised without increasing the radiating power, and hence the wireless range could be increased allowing for better performance in wireless communication link. These results and finding therefore could be used in the applications of wireless sensor networks where the need for power optimisation is very important. This analysis could be observed in figure 1, which shows clearly the advantages in introducing the multiple radiating elements to strengthen the SNR, and reducing the BER based on the derived formula (5). 


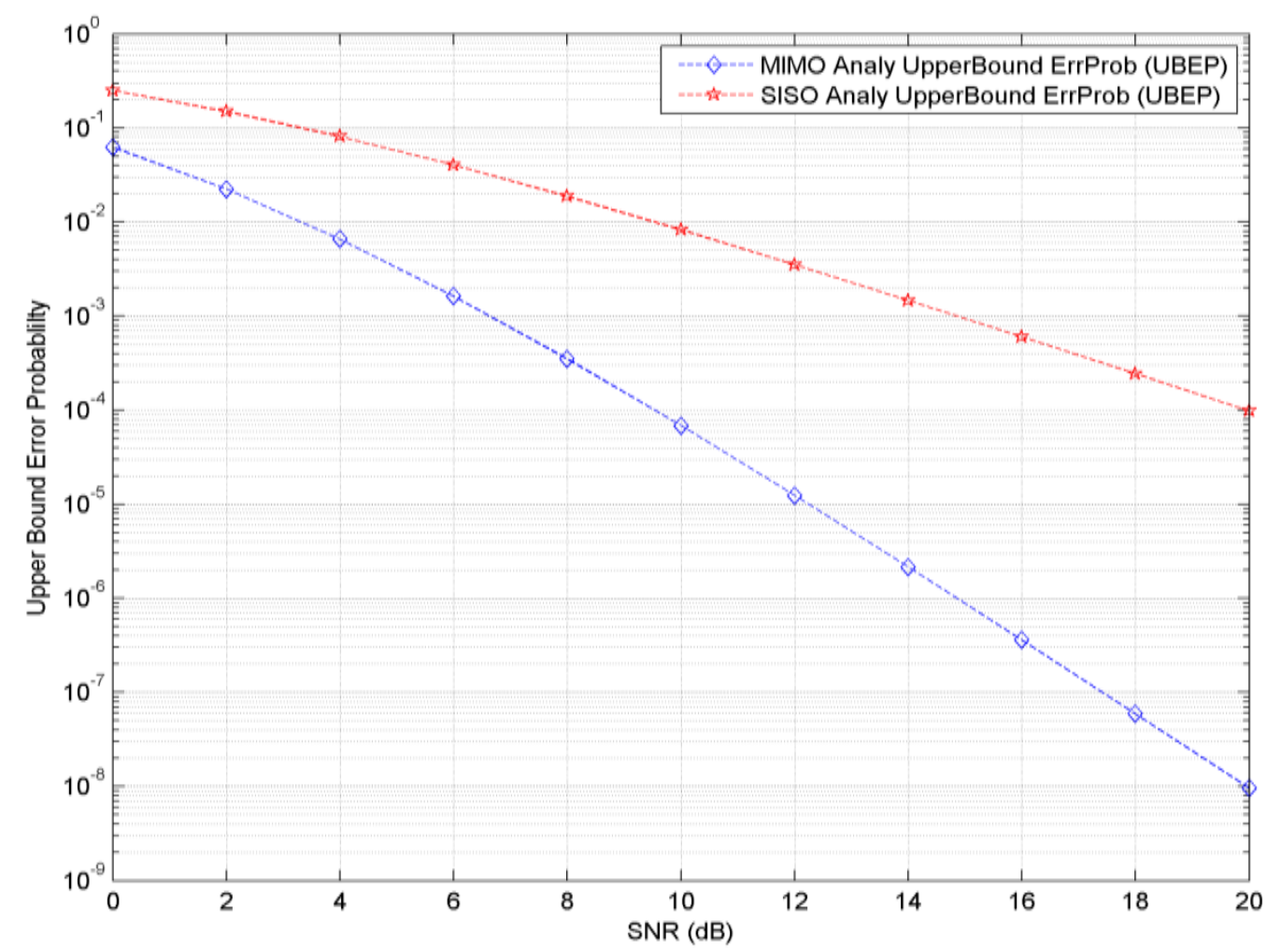

Figure (1): Analytical upper bound error probability comparison

\section{The Design of the Model Transmitting Structure}

The design architecture of the modulation scheme consists of two antennas, each of which contains dual QPSK constellations that convert the input stream into two sequences of complex symbols. Then, the pair of symbols within every branch was reordered by interchanging the In-phase components of this complex numbers. The output were then passed to a higher order modulation by incorporating the use of dual circular 8 points constellation maps in the name of 8-ary PSK modulation, and that facilitated high data rate transmission. In this concept, every branch represented a group of five bits, four of which relates to the dual complex symbols and the fifth bit was used for the control part. The process takes the interchanged complex symbols (coming from the dual QPSK symbols) and the fifth bit representing that particular group (G1 for the first branch, and G2 for the second branch). As every QPSK symbol would have four possibilities, and having two probabilities for the binary value of the fifth, then there are a total of eight expected signals for the modulation scheme that could be projected in the 8PSK constellation map. Figure 2 explains the core principle of the proposed scheme. 
In order to implement the scheme, the upper eight signal maps should be constructed according to the alignments. Hence, the proposed concept constructs the 8 points constellation so that all the points were positioned in the complex plane based on specific configuration. The constellations of these 8-ary PSK was designed so that there are two distinct distance metrics across the orthogonal In-phase and Quadrature domains on the four quadrants in the scatter diagram (Figure 3). The eight signal points were distributed across the two orthogonal domains based on two Euclidean metrics (d1, d2). Every point positioned on the same line of another point in the neighbouring quadrant on the In-phase and quadrature axes.

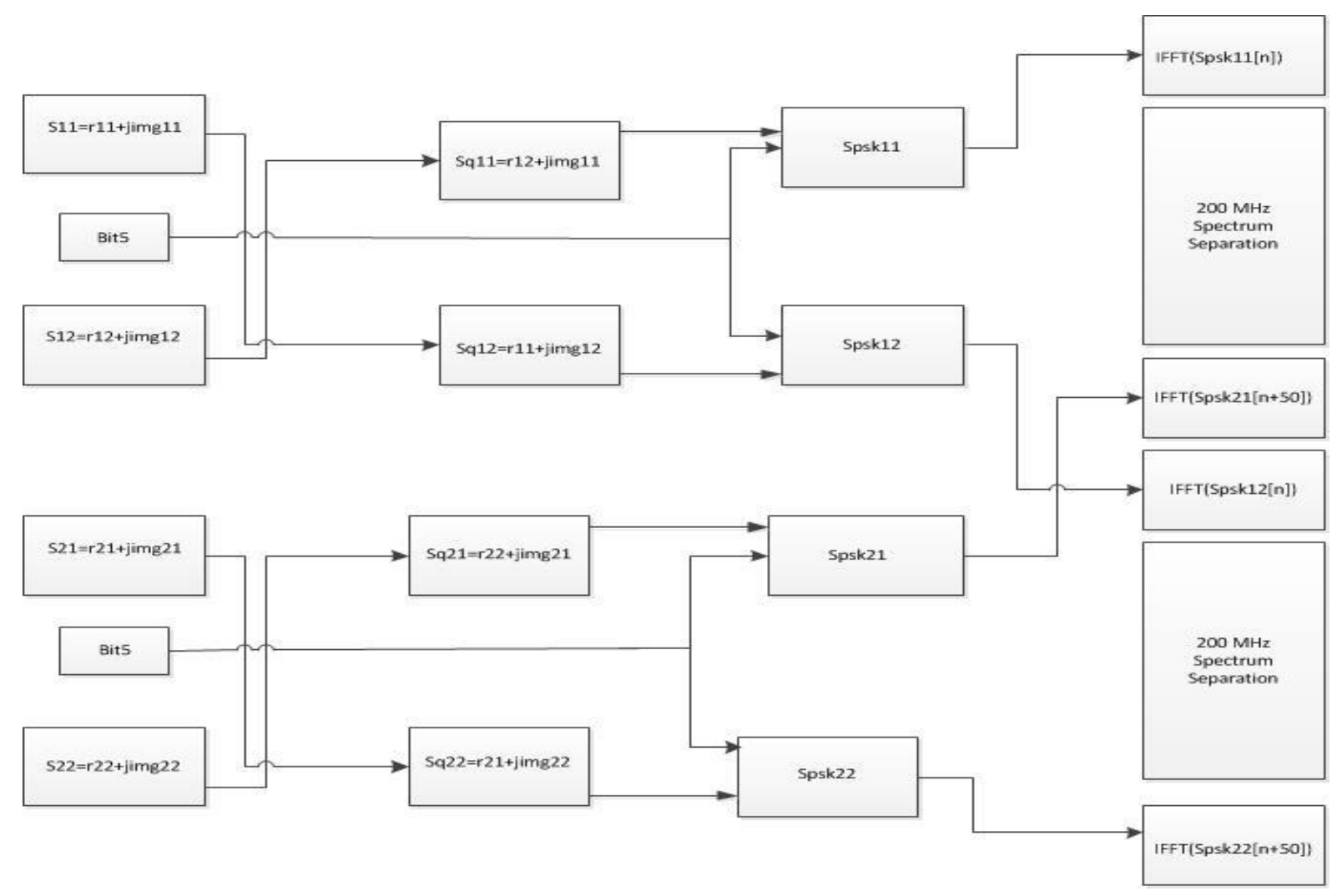

Figure (2): The transmitting configuration design across the two antennas 


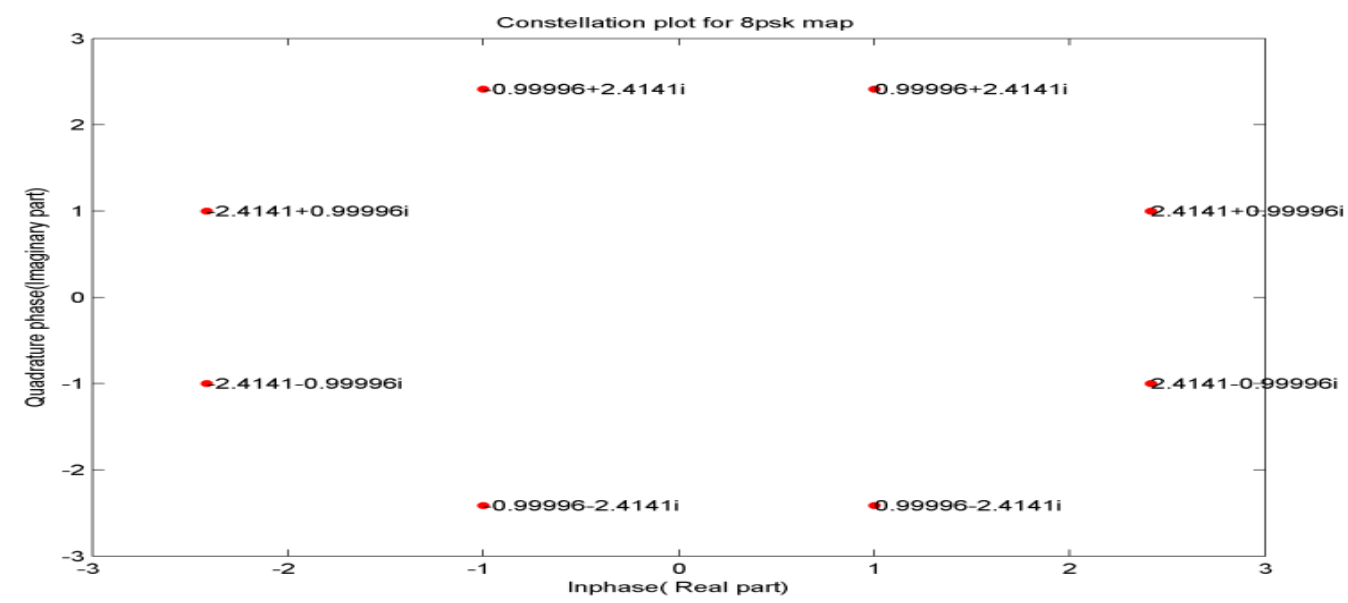

Figure (3): The two distance metrics across the 8 signal constellation points

In this 8-ary PSK modulation with constant amplitude modulation, the positions of symbols were rotated around the circle by $\frac{j 2 \pi}{16}$ (22.2 degrees), so as to ensure that only two distance metrics (d1, d2) from the origin across the real and imaginary axes in the complex plane contains the constellation points. These metric locations were used to position the four QPSK complex signals using the driving bit (b5) located in each of the two sub groups $(\mathrm{G} 1, \mathrm{G} 2)$. For the first branch, the two output complex symbols from the four point modulation (QPSK modulation) was multiplied by the distance metrics $(\mathrm{d} 1, \mathrm{~d} 2)$ so as to be transformed into PSK complex symbols ready to be distributed across the eight point maps. The first complex symbol was assigned to the first PSK constellation, while the second symbol representing the third and fourth bits within G1 was a located to the second constellation (Figure 4). The binary value of the fifth bit represents the most significant bit of the PSK symbol (three bits symbol), and was used as a reference to identify the particular signal point on the map in which these symbols represents. In a similar manner, these steps were applied across the second branch in parallel to achieve the spectral efficiency of the system. The original four quadrature symbols represented as follows

$$
\begin{aligned}
& \mathrm{s}_{q p s k_{1}^{G 1}}=x_{c(n)}^{G 1}+j x_{c(n)+50}^{G 1} \\
& \mathrm{~s}_{q p s k_{2}^{G 1}}=x^{G 1}{ }_{c(n)+1}+j x^{G 1}{ }_{c(n)+51} \\
& \mathrm{~s}_{q p s k_{1}^{G 2}}=x^{G 2}{ }_{c(n)}+j x^{G 2}{ }_{c(n)+50} \\
& \mathrm{~s}_{q p s k_{2}^{G 2}}=x^{G 2}{ }_{c(n)+1}+j x^{G 2}{ }_{c(n)+51} \\
& c(n)= \begin{cases}2 n & n \in\{0 \ldots .24\} \\
2 n+50 & n \in\{25 \ldots .49\}\end{cases}
\end{aligned}
$$

Where 
The metrics $\mathrm{d} 1$ and $\mathrm{d} 2$ were interchanged between the symbols based on b5 binary value. In the case of the first group, the following operation was done on the dual constellations.

For the first constellation

If $\mathrm{b} 5==0$

$\mathrm{s}_{p s k_{1}^{g 1}}(\mathrm{~m})=d 1 * \operatorname{real}\left(\mathrm{s}_{q p s k_{1}^{g 1}}\right)+d 2 * \operatorname{imag}\left(\mathrm{s}_{q p s k_{1}^{g 1}}\right)$

Else $(\mathrm{b} 5==1)$

$\mathrm{s}_{p s k_{1}^{g 1}}(\mathrm{~m})=d 2 * \operatorname{real}\left(\mathrm{s}_{q p s k_{1}^{g 1}}\right)+d 1 * \operatorname{imag}\left(\mathrm{s}_{q p s k_{1}^{g 1}}\right)$

For the second constellation

If $b 5==0$

$\mathrm{s}_{p s k_{2}^{g 1}}(\mathrm{~m})=d 2 * \operatorname{real}\left(\mathrm{s}_{q p s k_{2}^{g 1}}\right)+d 1 * \operatorname{imag}\left(\mathrm{s}_{q p s k_{2}^{g 1}}\right)$

Else

$\mathrm{s}_{p s k_{2}^{g 1}}(\mathrm{~m})=d 1 * \operatorname{real}\left(\mathrm{s}_{q p s k_{2}^{g 1}}\right)+d 2 * \operatorname{imag}\left(\mathrm{s}_{q p s k_{2}^{g 1}}\right)$

End 

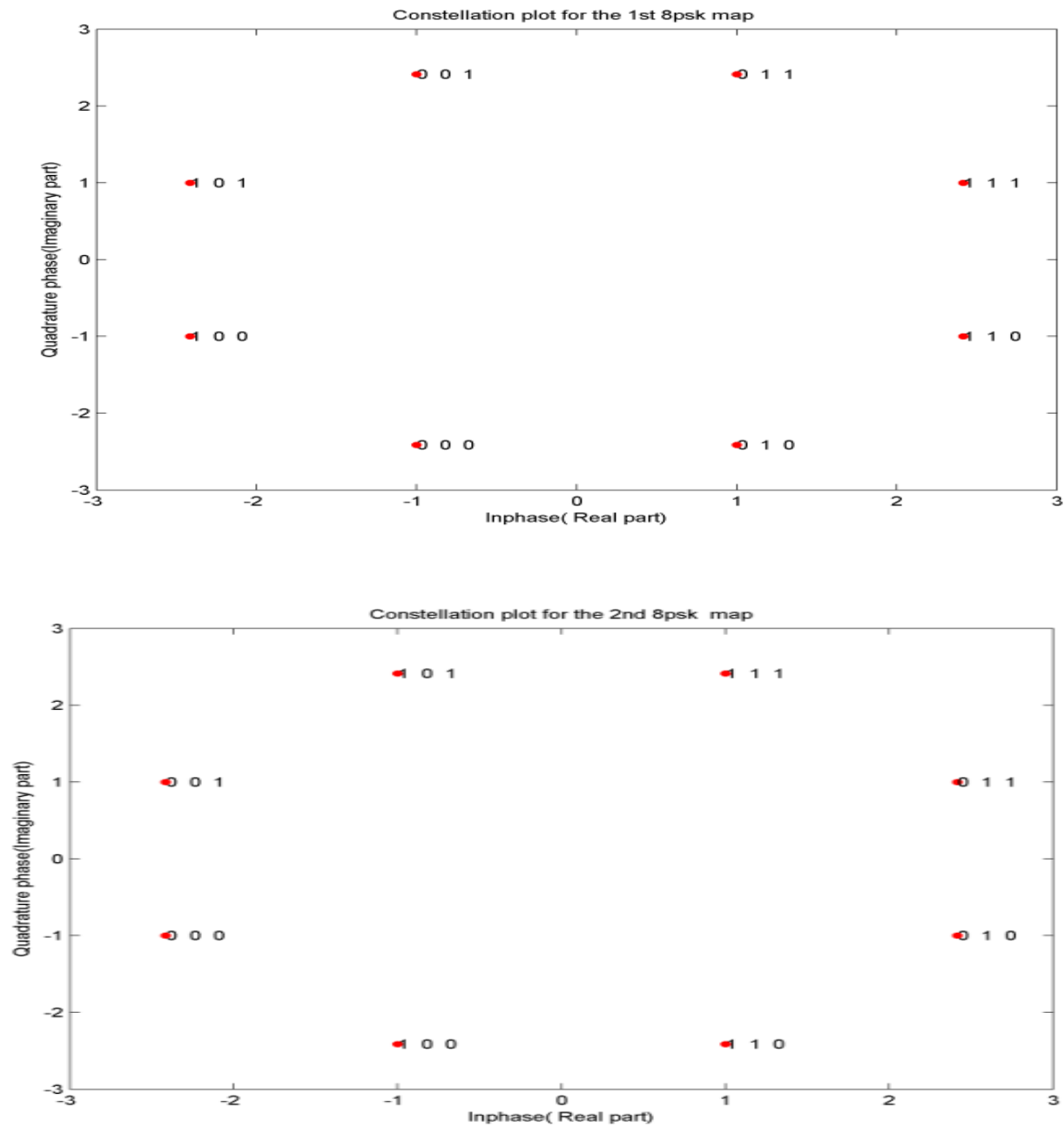

Figure (4): The constellation maps used for the dual 8-ary PSK symbols

The formulated PSK symbols were then allocated to different IFFT subcarriers with spatial variation and over $50 \mathrm{MHz}$ frequency separation. It is important to mention that, due to the use of MIMO and transmitting the dual symbols from every branch across two antennas, the actual frequency separation would be much greater, and insures maximum degree of separation in terms of the spectral fading across these symbols. The second transmitting branch would be subjected to the same process, which ensures compatibility with the proposed ECMA-368 standard. 


\section{The Receiving model structure}

The receiver architecture consists of two branches, and there were dual 8-PSK constellations in every branch. A cross interchanging was applied to the In-phase components with the four received complex symbols. This process was undertaken by taking the real amplitude of the first complex symbol within the second branch, and inserted in place of the In-phase component of the first symbol within the first receiver branch. In the same time, the amplitude of the first symbol within the first branch replaces the amplitude of the first complex symbol within the second receiving branch. In a similar process, the second received 8-PSK symbol within the first receiving branch was readjusted by replacing its real amplitude with its counterpart of the second symbol within the second receiving block. In across exchange, the second received 8-ary PSK complex symbol was modified by inserting the In-phase of the second symbol of the first branch in place of its real amplitude. Therefore, all the received signals carried by the four complex symbols were readjusted in this process. A combiner was added using the combining technique to strengthen the SNR by exploiting the diversity property exhibited in the received symbols, and figure (5) describes this design scheme. The recived signals at both dual receivers had been expressed by the following received symobls.

$$
\begin{aligned}
& R_{k(n)}^{p s k_{1}^{A 1}}(\mathrm{~m})=H_{k(n)}^{A 1} S_{k(n)}^{p s k_{1}^{A 1}}(\mathrm{~m})+\mathrm{z}_{k(n)}^{A 1} \\
& R_{k(n+50)}^{p s k_{2}^{A 1}}(\mathrm{~m})=H_{k(n+50)}^{A 1} S_{k(n+50)}^{p s k_{2}^{A 1}}(\mathrm{~m})+\mathrm{z}_{k(n+50)}^{A 1} \\
& R_{k(n)}^{p s k_{1}^{A 2}}(\mathrm{~m})=H_{k(n)}^{A 2} S_{k(n)}^{p s k_{1}^{A 2}}(\mathrm{~m})+\mathrm{z}_{k(n)}^{A 2} \\
& R_{k(n+50)}^{p s k_{2}^{A 2}}(\mathrm{~m})=H_{k(n+50)}^{A 2} S_{k(n+50)}^{p s k_{2}^{A 2}}(\mathrm{~m})+\mathrm{z}_{k(n+50)}^{A 2}
\end{aligned}
$$

Where $H_{k(n)}^{i}$ and $H_{k(n+50)}^{i}$ were the channel spectrums for the first anad second sections of the ith receiver.

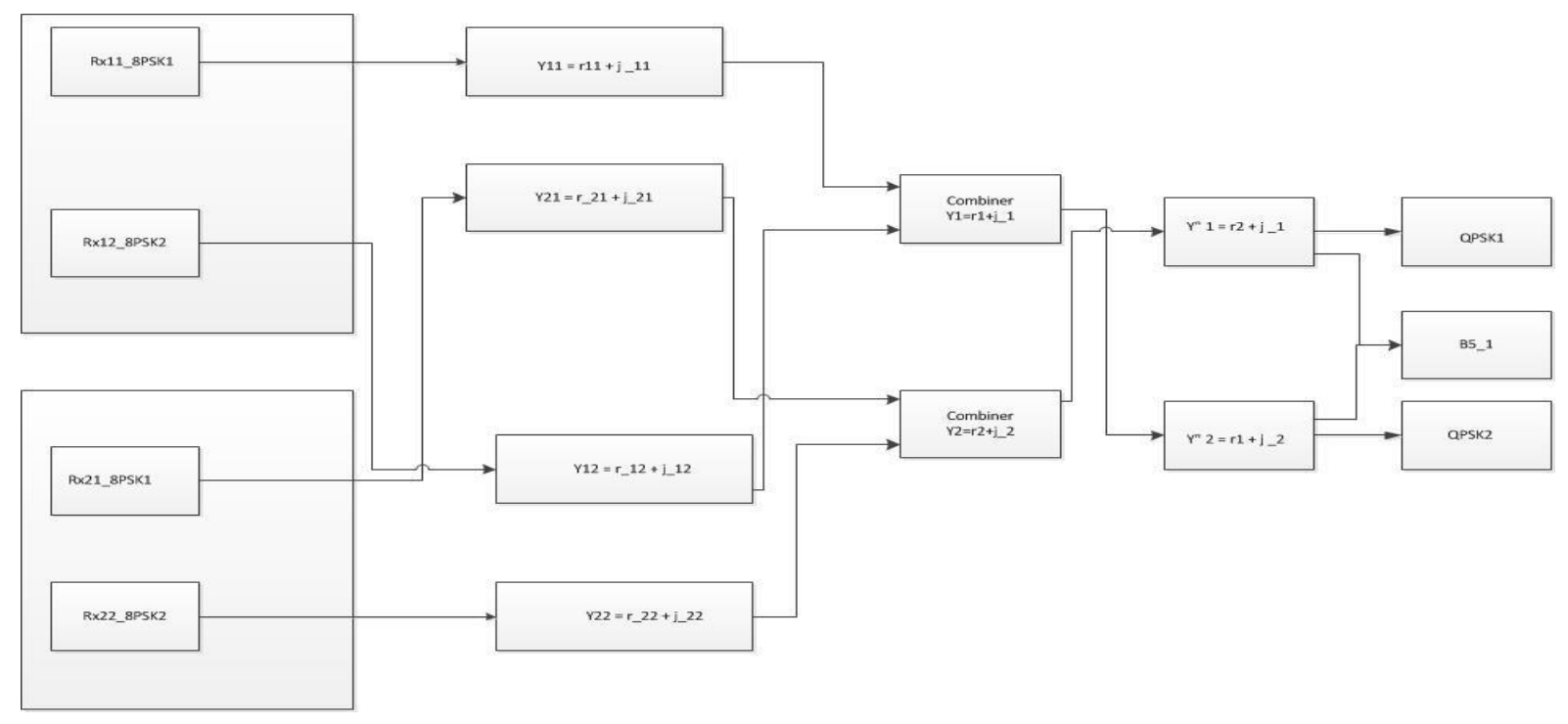

Figure (5): The design scheme for the dual receiver's structure

\section{Performance analyses}


In this section, our model (The proposed Spatial Diversity MIMO Model) and its behaviour was evaluated including how the configuration make use of the full frequency and spatial diversities in increase the coverage area of the system. The simulation process and its observed results have been carried out using the MATLAB environment. The results were averaged over 1000 packets in terms of the SNR metric. The behaviour of the system was analysed when implementing two transmitters and two receivers in the proposed Spatial Diversity MIMO Model configuration for SensApp (Sensor Applications). The results were then compared to the single antenna DC 32-QAM based on two 8-ary PSK constellations. The IEEE 82.15.3a standard channel proposal was used in the simulation with CM1 referring to the LOS channel model and CM2 containing the medium property for the NLOS modelling. The simulated system was a Multiband OFDM transmission having 128FFT subcarrier frequency tones with a bandwidth of $528 \mathrm{MHz}$ for each sub-band. As the proposed aim was to increase the receiving signal energy, and over shadowing the throughput. Therefore, the model had replaced the multiplexing technique with the use of diversity to strengthen the SNR at the receivers. The first figure (6) showed an error performance comparison between the proposed Spatial Diversity MIMO Model for SensApp, the original designed model (single antenna DC32QAM-based on 8PSK model), and the Spatial Multiplexing MIMO model Configuration. The figure clearly shows the performance improvement the proposed Spatial Diversity MIMO model achieved in comparison to the other concepts. Figure (7) on the other hand, shows the relation between the coverage area and packet error rate, and indicates the increase in the wireless range of the model and its design credentials. It is very clear from the figure that our proposed model implementing dual antennas outperform the standard single model transmission. 


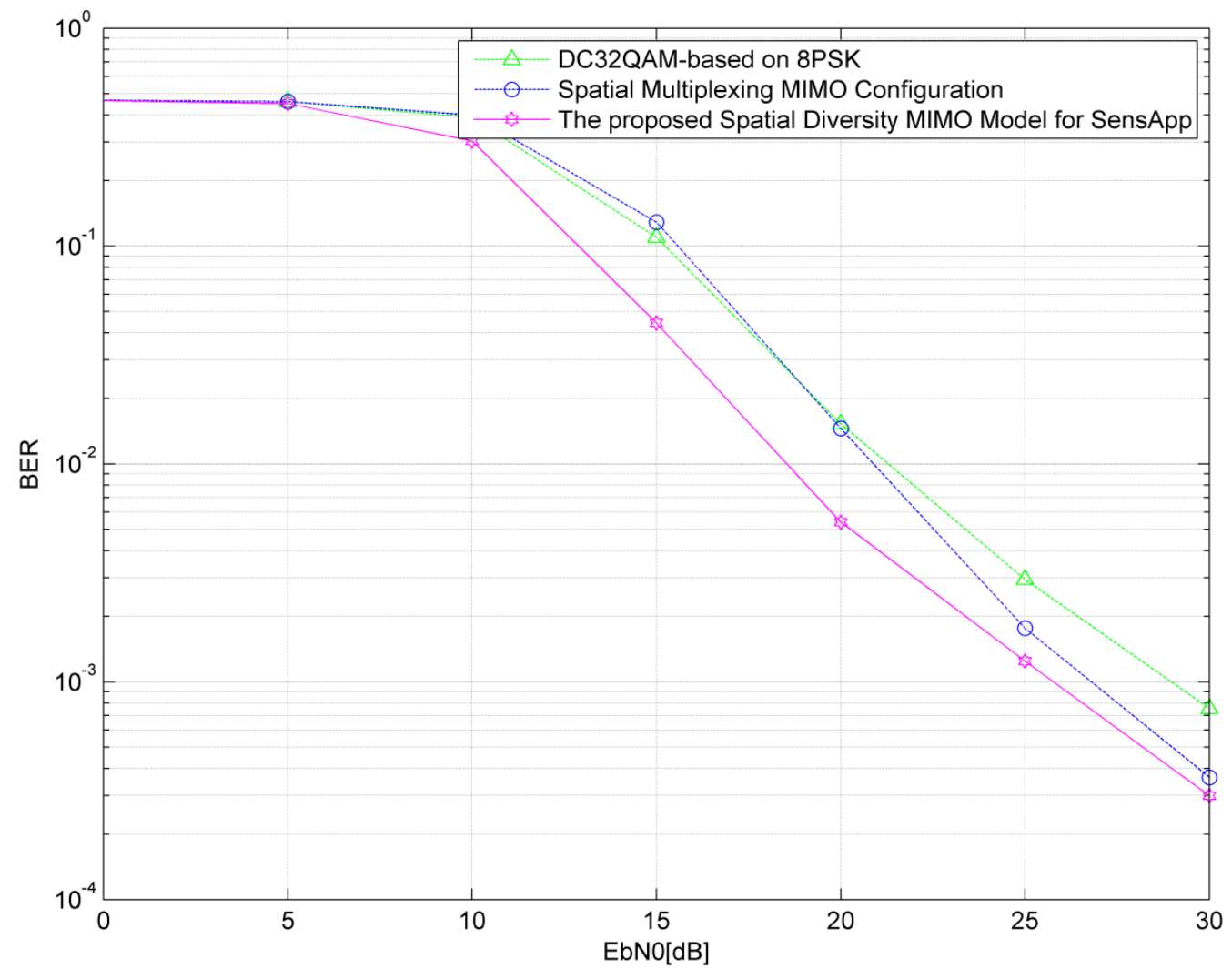

Figure (6): BER comparison between the proposed models 


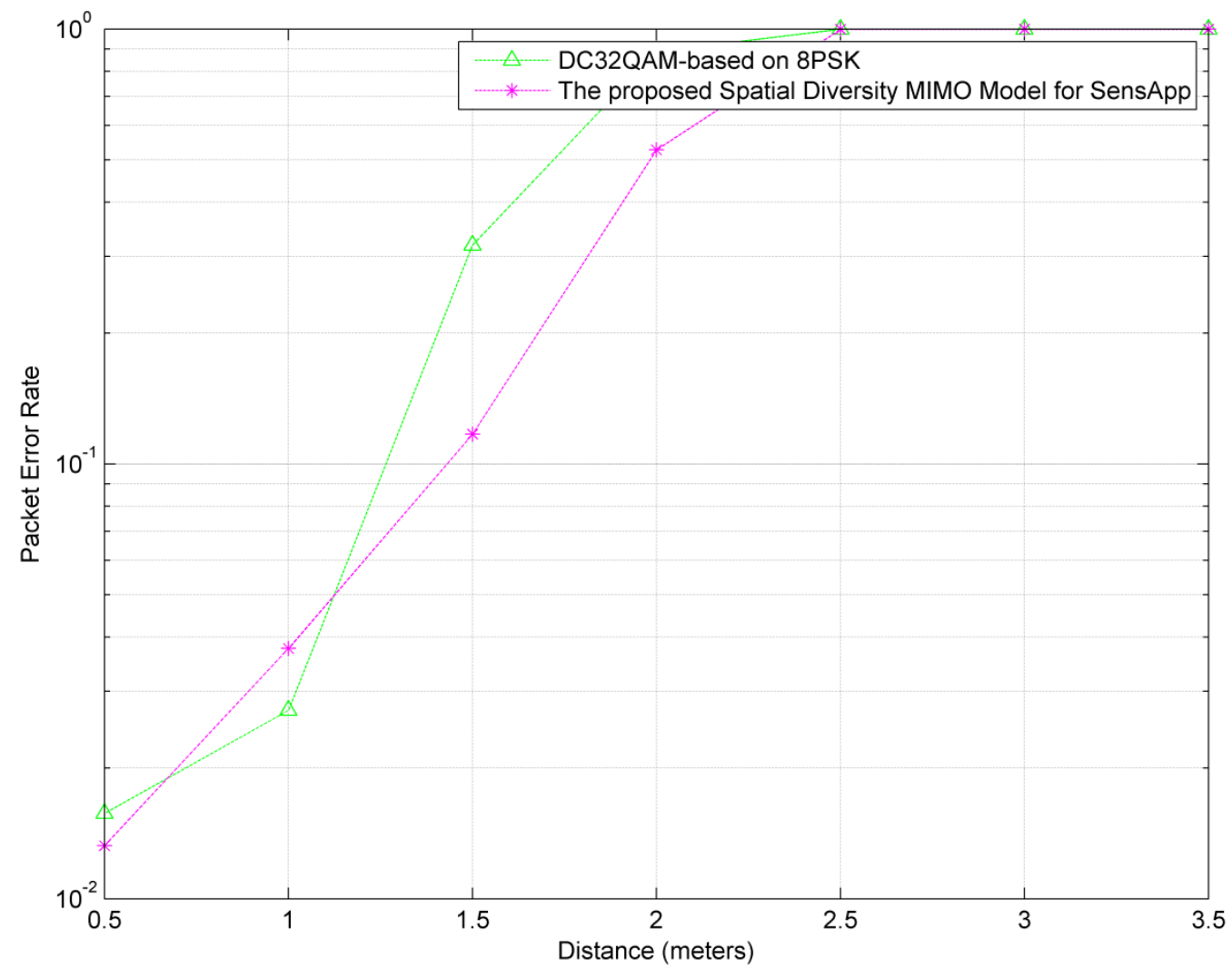

Figure (7): Performance comparison of the models over coverage area

\section{Conclusions}

In this paper, a design for a MIMO model was developed that facilitates an increase in the coverage area for wireless sensor network applications. The proposed method makes use of the spatial diversity, frequency selectivity and fading variation across the spectrum to improve the SNR at the receivers. The novelty of this proposal is achieved using multiple spatial diversity that enables transmitting at a higher order of modulation whilst decoding at a lower order modulation scheme. This process improves the SNR and reduces the error rate at the receivers by reducing the decoding search space, and expanding the Euclidean metric between adjacent signal points in the receiving constellation map. It also provides a framework for future system development. Finally, simulation results incorporating a standardised channel model have shown improvements in the BER for the MIMO DC 32-QAM model when compared to a standard classical model. 


\section{REFERENCES}

1) B. A. Cetiner, E. Akay, E. Sengul, and E. Ayanoglu,"A MIMO System With Multifunctional Reconfigurable Antennas," Antennas and Wireless Propagation Letters, IEEE, vol. 5, pp. 463-466, 2006.

2) L.Junsheng, M. Ghavami, C. Xiaoli, B. Allen, and W. Malik, "Diversity Analysis of Multi-Antenna UWB Impulse Radio Systems with Correlated Propagation Channels," in Wireless Communications and Networking Conference, 2007.WCNC 2007. IEEE, 2007,pp. 1593-1598.

3) A. Stephan, J. F. Helard, and B. Uguen, "MIMO UWB Systems Based on Linear Precoded OFDM for Home Gigabit Applications," in Global Telecommunications Conference, 2008. IEEE GLOBECOM 2008. IEEE, 2008, pp. 1-6.

4) J. Paulraj, D. A. Gore, R. U. Nabar, and H. Bolcskei, "An overview of MIMO communications - a key to gigabit wireless," Proceedings of the IEEE, vol. 92, pp.198-218, 2004.

5) L. Zhiwei, P. Xiaoming, F. Chin, and P. Khiam Boon, "Enhanced MB-OFDM UWB system with multiple transmit and receive antennas," in Ultra-Wideband, 2009. ICUWB 2009. IEEE International Conference on, 2009, pp. 793-797.

6) A. Batra, J. Balakrishnan, A. Dabak, "Multi-band OFDM: a new approach for UWB,"Circuits and Systems, ISCAS '04. Proceedings of the 2004 International Symposium on,vol.5, no., pp. V-365- V-368 Vol.5, 23-26 May 2004.

7) F. Molisch, J. R. Foerster, and M. Pendergrass, "Channel models for ultrawideband personal area networks," Wireless Communications, IEEE, vol. 10,pp. 14-21, 2003.

8) "Standard ECMA-368: High rate ultra wideband PHY and MAC standard," ECMA, First Edition, Dec. 2005.

9) A. Batra and J. Balakrishnan, "Improvements to the multi-band ofdm physical layer," in Consumer Communications and Networking Conference, 2006. CCNC 2006. 3rd IEEE, 2006, pp. 701-705.

10) R. Hyun-Seok, L. Jun-Seok, and C. G. Kang, "BER analysis of dual-carrier modulation (DCM) over Rayleigh fading channel," in Ultra Modern Telecommunications and Control Systems and Workshops (ICUMT), 2010 International Congress on, 2010, pp. 717-721.

11) T. H. Nguyen, T. H. Nguyen, V. T. Tran, B. G. Truong Vu, and V. D. Nguyen, "A scheme of Dual Carrier Modulation with Soft-decoding for MB-OFDM MIMO systems," in Advanced Technologies for Communications (ATC), 2011 International Conference on, 2011, pp. 220-223.

12) Y. Runfeng and R. Sherratt, "Enhancing MB-OFDMthroughput with dual circular 32-QAM," ConsumerElectronics, IEEE Transactions on, vol.54, pp. 1640-1646,2008.

13) V. Tarokh, N. Seshadri, and A.R. Calderbank, "Space-time codes for high data rate wireless communication: performance criterion and code construction," IEEE Trans.Info Theory, vol. 44, no. 1, pp. 7 44-7 65, Mar. 1998.

14) I. S. Gradshteyn and I. M. Ryzhik, Table of Integrals, Series, and Products, 5th ed. San Diego, CA: Academic, 1994. 